\title{
Mapping the Antibody Repertoires in Ferrets with Repeated Influenza A/H3 Infections: Is Original Antigenic Sin Really "Sinful"?
}

Tal Einav ${ }^{1, *, \dagger}$, Martina Kosikova ${ }^{2, *}$, Peter Radvak ${ }^{2}$, Yuan-Chia Kuo ${ }^{2}$, Hyung Joon Kwon ${ }^{2}$, Hang Xie ${ }^{2, \dagger}$

${ }^{1}$ Basic Sciences Division and Computational Biology Program, Fred Hutchinson Cancer Research Center, Seattle, Washington, United States of America. ${ }^{2}$ Laboratory of Respiratory Viral Diseases, Division of Viral Products, Office of Vaccines Research and Review, Center for Biologics Evaluation and Research, United States Food and Drug Administration, Silver Spring, Maryland, United States of America.

* These authors contributed equally to this work.

†Correspondence to: teinav@fredhutch.org, hang.xie@fda.hhs.gov

\begin{abstract}
The influenza-specific antibody repertoire is continuously reshaped by infection and vaccination. The host immune response to contemporary viruses can be redirected to preferentially boost antibodies specific for viruses encountered early in life, a phenomenon called original antigenic $\sin (\mathrm{OAS})$ that is suggested to be responsible for diminished vaccine effectiveness after repeated vaccination. In this study, we used a new computational tool called Neutralization Map to determine the hemagglutination inhibition profiles of individual antibodies within ferret antisera elicited by repeated influenza A/H3 infections. Our results suggest that repeated infections continuously reshape the ferret antibody repertoire, but that a broadly neutralizing antibody signature can nevertheless be induced irrespective of OAS. Overall, our study offers a new way to visualize how immune history shapes individual antibodies within a repertoire, which may help inform future vaccine design.
\end{abstract}

\section{Keywords}

Mapping antibody repertoires; antibody tracking; original antigenic sin; immune imprinting; repeated influenza exposures; ferret reinfection model; broadly neutralizing antibody; influenza A $\mathrm{H} 3 \mathrm{~N} 2$ virus

\section{Introduction}

Rapidly evolving pathogens such as influenza frequently change their antigenicity in order to escape the host immune system, and the emergence of antigenically drifted strains necessitates the annual update of seasonal influenza vaccine components. Despite efforts to forecast which strain(s) will be most prevalent, a suboptimal or mismatched vaccine strain may occasionally be selected for vaccine production, resulting in reduced protection. ${ }^{1-4}$ In the US, influenza vaccine effectiveness in the past decades has fluctuated significantly from $10 \%$ in the $2004-2005$ season $^{1}$ to $60 \%$ in the 2010-2011 season (https://www.cdc.gov/flu/vaccines-work/effectivenessstudies.htm $).{ }^{5}$ While vaccine mismatch directly accounts for this low efficacy, pre-existing host immunity also influences vaccine performance. ${ }^{3,6-12}$ 
An individual's exposure history, acquired through recurrent infections and/or vaccinations, shapes their unique antibody repertoire and influences their response to newly emerging influenza viruses. ${ }^{6,10-19}$ For example, several recent studies have reported that vaccine effectiveness is negatively correlated with vaccination frequency, with lower efficacy seen in more frequent vaccinees. ${ }^{1,20-25}$ While the exact mechanisms remain unknown, a suggested confounding factor is original antigenic sin (OAS) - a phenomenon where immune memory is recalled toward strains encountered early in life rather than to evolved viruses. ${ }^{26} 9,25,27-29$ On the other hand, residual antibodies from prior exposures may grant subsequent protection against viruses with similar antigenicity. ${ }^{15-17,29}$ These reports provide a glimpse of the complex interplay between prior and current immunity, highlighting the influence of immune imprinting that must be addressed in the field of vaccinology.

In this work, we profiled the hemagglutination inhibition (HAI) responses in ferrets after repeated influenza $\mathrm{A} / \mathrm{H} 3$ infections and mapped the $\mathrm{HAI}$ antibodies elicited using a computational tool called Neutralization Map that characterizes antibody inhibition patterns. ${ }^{30}$ By tracking the progression of HAI antibodies following a series of infections, we demonstrated that repeated exposure to influenza can guide antibodies towards specific inhibition profiles irrespective of OAS.

\section{Results}

\section{Sequential infections extended antibody cross-reactivity and induced broadly-neutralizing antibodies}

We first conducted a sequential infection experiment in which seronegative ferrets were exposed to four influenza A/H3 viruses: A/Uruguay/716/2007 (Uruguay 2007, denoted as $\mathrm{V}_{1}$ throughout this work), A/Texas/50/2012 (Texas 2012, V2), A/Switzerland/9715293/2013 (Switzerland 2013, $\mathrm{V}_{3}$ ), and A/Hong Kong/4801/2014 (Hong Kong 2014, $\mathrm{V}_{4}$ ) as previously reported. ${ }^{6}$ We tracked the progression of antibodies developed after infection with $\mathrm{V}_{1}$ alone, followed by infection with the second $\left(\mathrm{V}_{1} \rightarrow \mathrm{V}_{2}\right)$, third $\left(\mathrm{V}_{1} \rightarrow \mathrm{V}_{2} \rightarrow \mathrm{V}_{3}\right)$, and fourth virus $\left(\mathrm{V}_{1} \rightarrow \mathrm{V}_{2} \rightarrow \mathrm{V}_{3} \rightarrow \mathrm{V}_{4}\right)$, to demonstrate how the antibody repertoire was shaped by recurring exposures. As shown in Figure 1A, Uruguay $2007\left(\mathrm{~V}_{1}\right)$ infection elicited $\mathrm{V}_{1}$-specific ferret HAI titers with limited cross-reactivity towards viruses that emerged before 2005 or after 2007. Following each sequential infection with $\mathrm{V}_{2}, \mathrm{~V}_{3}$, and $\mathrm{V}_{4}$, the cross-reactivity of ferret antisera gradually extended with geometric mean titers (GMTs) $\geq 80$ against all A/H3 viruses in the panel except A/Philippines/2/1982 (Philippine 1982) that had disappeared from circulation more than three decades earlier (Figure 1B-1D). 

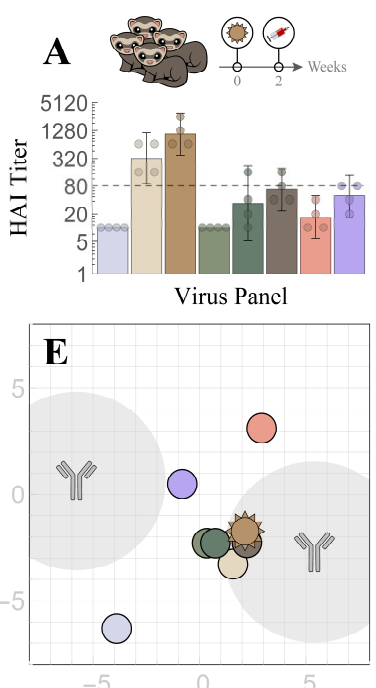

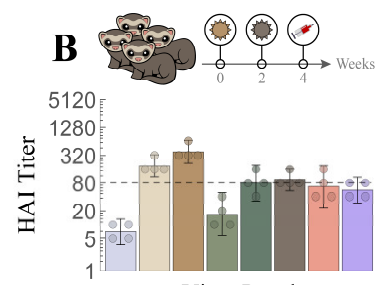

Virus Pancl

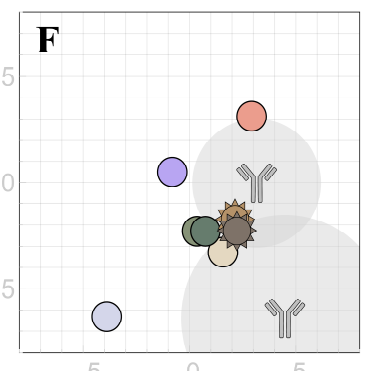

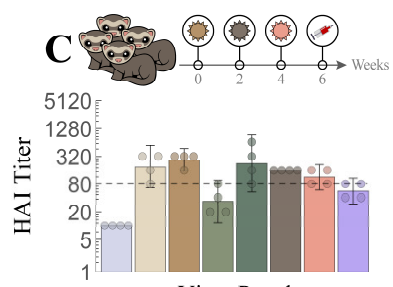

Virus Pancl

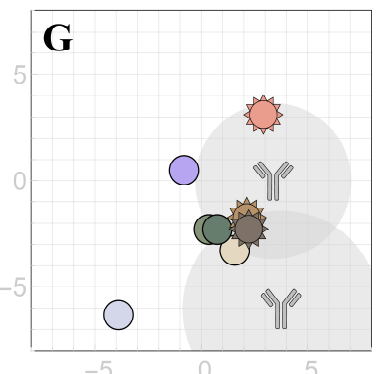

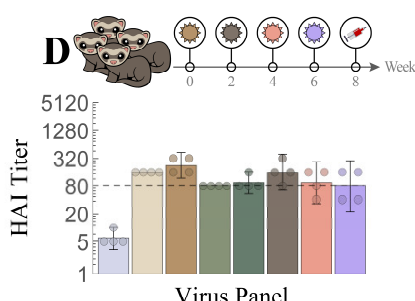

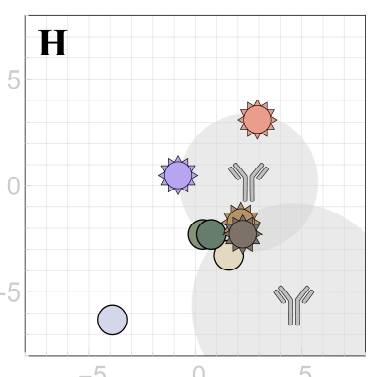

H3N2 Virus $\square$ A/Philippines/2/1982
$\square$ A/Wisconsin/67/2005 $\square$ A/Wisconsin/67/2005
$\square$ A/Uruguay/716/2007 A/Perth/16/2009 A/Victoria/361/2011 A/Texas $/ 50 / 2012$ $\square$ A/Switzerland/9715293/2013 $\square$ A/Hong Kong/4801/2014 Decomposition -Antibody HAI Titer $\geq 80$ 0 Virus in Panel Infection Strain Map Distance f $d \bigcirc$ HAI Titer $=3000 / 2^{d}$

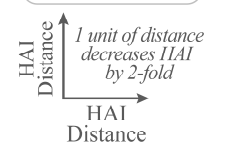

Figure 1. Tracking individual antibodies using hemagglutination inhibition (HAI) responses in ferrets during each stage of four sequential H3N2 infections. (A-D) Naïve ferrets were sequentially infected with $\mathrm{V}_{1}=$ Uruguay $2007, \mathrm{~V}_{2}=$ Texas $2012, \mathrm{~V}_{3}=$ Switzerland 2013 , and $\mathrm{V}_{4}=$ Hong Kong 2014 . HAI titers are shown for each step of the infection: (A) $\mathrm{V}_{1}$, (B) $\mathrm{V}_{1} \rightarrow \mathrm{V}_{2}$, (C) $\mathrm{V}_{1} \rightarrow \mathrm{V}_{2} \rightarrow \mathrm{V}_{3}$, and (D) $\mathrm{V}_{1} \rightarrow \mathrm{V}_{2} \rightarrow \mathrm{V}_{3} \rightarrow \mathrm{V}_{4}$. Individual HAI titers are presented from four ferrets (points) with geometric means (bar graphs) and $95 \%$ confidential intervals (error bars). (E-H) These HAI measurements were decomposed to determine the individual antibodies elicited after infection with (E) $V_{1}$ followed by $(F) V_{2},(G) V_{3}$, and (H) $V_{4}$. Each antibody signature (gray) is predicted to have an HAI titers $\geq 80$ against any virus within the gray circle, with the size of this circle proportional to the fractional composition of the antibody in the serum [SI Methods]. An antibody-virus distance $d$ denotes an HAI titer of $3000 / 2^{d}$. Virus coordinates were previously determined using a panel of monoclonal antibodies. ${ }^{30}$

To track antibody development throughout these four infections, we decomposed the HAI GMTs (Figure 1A-1D) using neutralization maps (Figure 1E-1H). On these maps, the positions of the eight influenza A/H3 viruses (identified by virus icons and solid dots) were previously determined using neutralizing data from a panel of monoclonal antibodies. ${ }^{30}$ Using these virus coordinates, the ferret antisera were computationally dissected to determine the number and location of antibody signatures that best match ferret HAI titers [SI Methods and Figures S1 and S2]. Each antibody signature may represent an amalgam of antibodies with similar inhibition profiles, although sufficiently distinct antibodies are decomposed as separate antibody signatures. The neutralization maps only show the strongest detectable antibody signatures that likely overwhelm the inhibition from weaker antibodies placed further away on the map. The Euclidean distance $d$ between an antibody signature and a virus on the map translates into an HAI titer of $3000 / 2^{d}$, with potent antibodies lying near viruses against which they have a high titer. The gray regions surrounding each antibody signature indicate an HAI titer $\geq 80$ against any virus that lies within (and we refer to such viruses as strongly inhibited).

As shown in Figure 1E, infection by $V_{1}$ (Uruguay 2007) resulted in one "specific" antibody signature that strongly inhibited $V_{1}$ and two nearby viruses - A/Wisconsin/67/2005 (Wisconsin 2005) and Texas 2012 - as well as another "non-specific" antibody signature that weakly inhibited all $\mathrm{A} / \mathrm{H} 3$ viruses in the testing panel. With each subsequent infection $\left(\mathrm{V}_{1} \rightarrow \mathrm{V}_{2}\right.$, 
$\mathrm{V}_{1} \rightarrow \mathrm{V}_{2} \rightarrow \mathrm{V}_{3}$, and $\mathrm{V}_{1} \rightarrow \mathrm{V}_{2} \rightarrow \mathrm{V}_{3} \rightarrow \mathrm{V}_{4}$ ), the specific antibody moved by 1-2 units in a manner that kept all infection strains strongly inhibited (within the gray circular regions surrounding each antibody Figure 1E-1H). Moreover, after the fourth infection, the GMTs across the entire virus panel were within 4-fold of one another (except for the older Philippines 1982 strain), indicating extended cross-reactivity of ferret antisera (Figure 1B-1D). While these maps depicted the average response of four ferrets, the individual maps of ferrets \#1-4 in this cohort showed the same antibody trajectories (Figure S3). These progressional maps collectively suggest that a broadly-neutralizing antibody signature, which we define as an antibody with HAI titer $\geq 80$ against all infection strains, can be guided into place by sequential exposures.

\section{Prior influenza exposures resulted in OAS and changed the inhibition profiles of elicited antibodies}

We next compared the HAI response of ferrets infected with $\mathrm{V}_{4}$ alone with the responses elicited after one $\left(\mathrm{V}_{3} \rightarrow \mathrm{V}_{4}\right)$, two $\left(\mathrm{V}_{2} \rightarrow \mathrm{V}_{3} \rightarrow \mathrm{V}_{4}\right)$, or three prior infections $\left(\mathrm{V}_{1} \rightarrow \mathrm{V}_{2} \rightarrow \mathrm{V}_{3} \rightarrow \mathrm{V}_{4}\right)$ to assess how exposure history affected the HAI antibody response to the final infection by $\mathrm{V}_{4}$. As shown in Figure 2A, infection by Hong Kong $2014\left(\mathrm{~V}_{4}\right)$ alone elicited higher HAI titers toward itself than to the other viruses in the panel. With additional prior exposures, ferret antisera always exhibited lower HAI GMTs toward $\mathrm{V}_{4}$ than to any of the earlier infection strains (Figure 2B-2D): for example, the $\mathrm{V}_{3} \rightarrow \mathrm{V}_{4}$ infections elicited an HAI GMT of 34 toward $\mathrm{V}_{4}$ compared to the GMT of 135 toward $V_{3}$, a typical OAS response that was also seen in Figure 1B-1D.
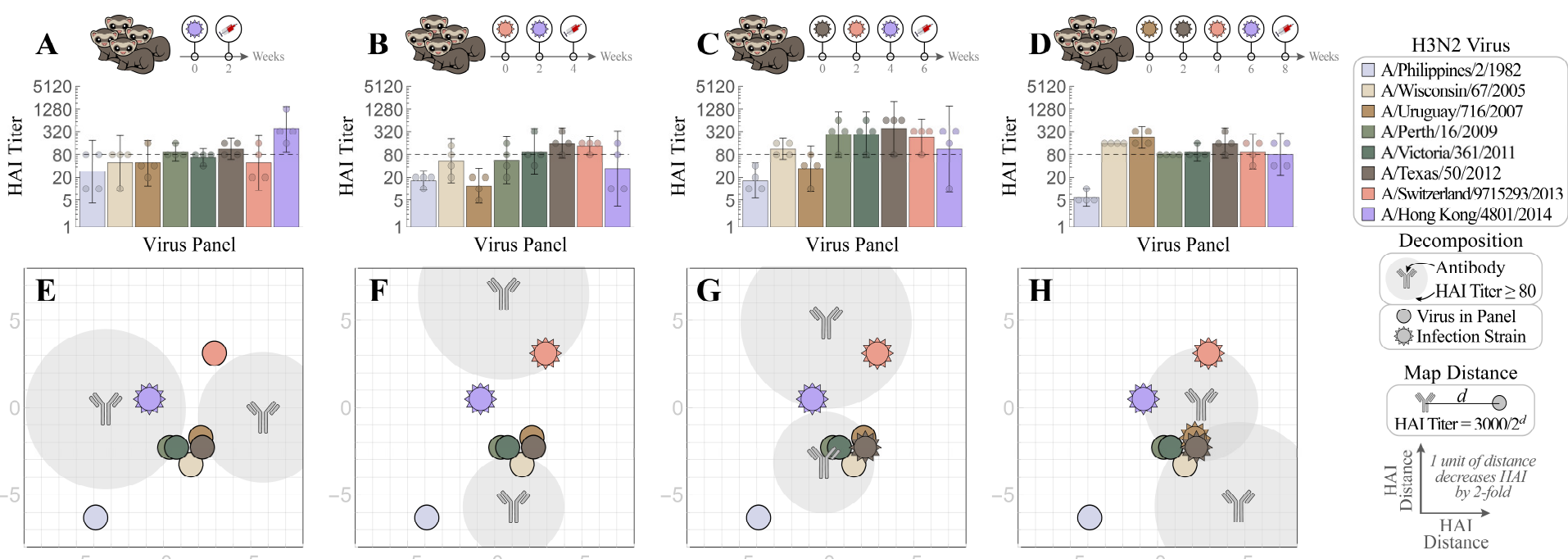

Figure 2. Mapping how exposure history shapes the ferret antibody response. Naïve ferrets were infected with (A) $\mathrm{V}_{4}=$ Hong Kong 2014 , or with prior exposure to (B) $\mathrm{V}_{3}=$ Switzerland 2013 , (C) $\mathrm{V}_{2}=$ Texas 2012 , and (D) $\mathrm{V}_{1}=$ Uruguay 2007 . All antisera were analyzed via HAI after the final infection with Hong Kong 2014. Individual HAI titers are shown from four ferrets (points) and geometric means (bar graphs) with 95\% confidential intervals (error bars). (E-H) Each set of HAI measurements was decomposed to determine the number of antibodies and their inhibition profiles. Each antibody signature (gray) is predicted to have an HAI titers $\geq 80$ against any virus within the gray circle, with the size of this circle proportional to the fractional composition of the antibody in the serum [SI Methods]. An antibody-virus distance $d$ denotes an HAI titer of $3000 / 2^{d}$. 
To discern how prior exposures impacted subsequent antibody development, we decomposed the HAI GMTs on the neutralization maps (average response shown in Figure 2E-2H and individual responses shown in Figure $\mathrm{S} 4$ ). Upon infection by $\mathrm{V}_{4}$ alone, one specific antibody signature emerged that strongly inhibited $\mathrm{V}_{4}$ along with a non-specific antibody signature that displayed weak inhibition against the infection strain (Figure 2E). This same pattern was also observed in ferrets singly infected by $\mathrm{V}_{1}, \mathrm{~V}_{2}$ or $\mathrm{V}_{3}$ (Figures 1-3 and S5). With each additional prior infection, OAS could be seen on the maps by noting that $\mathrm{V}_{4}$ lay further from the center of the gray antibody circles than the earlier infection strains, indicating a lower HAI response (Figure 2G$2 \mathrm{H})$. Nevertheless, following the $\mathrm{V}_{1} \rightarrow \mathrm{V}_{2} \rightarrow \mathrm{V}_{3} \rightarrow \mathrm{V}_{4}$ infections, ferrets developed antibodies that inhibited not only all four infection strains but also the other $\mathrm{H} 3$ viruses in the panel except Philippines 1982. Taken together, these results suggest that prior exposures affect the antibody response, but that a broadly-neutralizing antibody signature can be induced by repeated exposures, even in the presence of OAS.
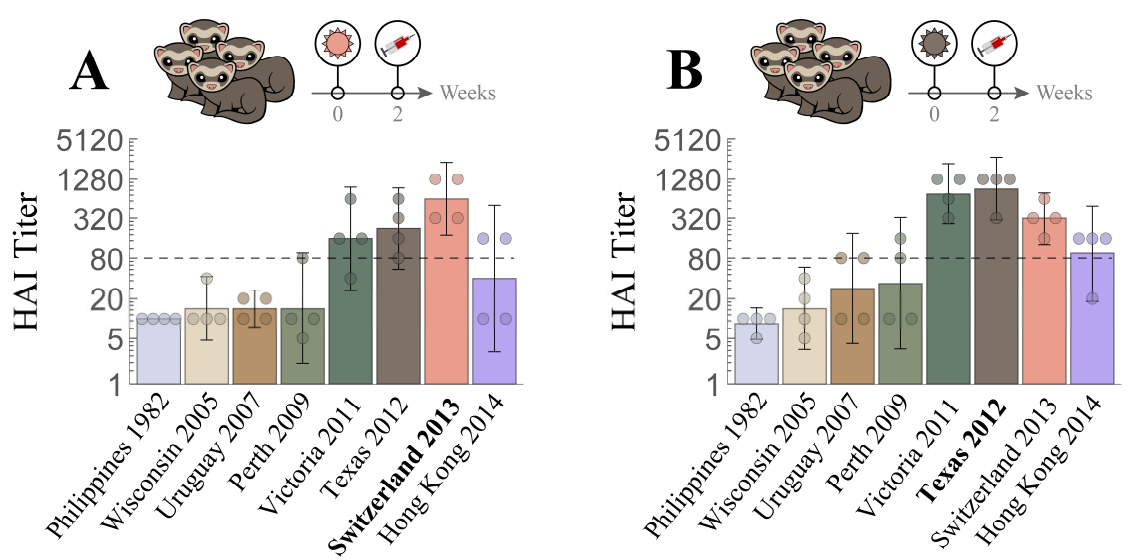

Figure 3. Cross-reactive hemagglutination inhibition (HAI) responses in ferrets with single H3N2 infection. Naïve ferrets were singly infected with either (A) $V_{3}=$ Switzerland 2013 or (B) $V_{2}=$ Texas 2012 . Individual HAI titers from four ferrets (points) and geometric means (bar graphs) are shown with 95\% confidential intervals (error bars).

\section{The antibody profile elicited by sequential infections is strain-specific}

We noticed that infection by $\mathrm{V}_{1} \rightarrow \mathrm{V}_{2}$ resulted in potent inhibition (HAI $\geq 80$ ) against both $\mathrm{V}_{1}$ and $\mathrm{V}_{2}$ (Figure $1 \mathrm{~F}$ ), whereas infection by $\mathrm{V}_{3} \rightarrow \mathrm{V}_{4}$ resulted in antibodies that strongly inhibited $\mathrm{V}_{3}$ but not $\mathrm{V}_{4}$ (Figure $2 \mathrm{~F}$ ). To delve more deeply into this discrepancy, we analyzed the individual response of each ferret in these cohorts. Infection by $\mathrm{V}_{1}$ alone generated homologous HAI titers $\geq 640$, whereas $V_{1} \rightarrow V_{2}$ led to HAI titers $\geq 80$ against both infection strains (Figure 4A-4D). In contrast, while infection by $\mathrm{V}_{3}$ alone generated homologous HAI titers $\geq 320$, the subsequent infection $\mathrm{V}_{3} \rightarrow \mathrm{V}_{4}$ resulted in different HAI responses in Ferrets \#5 and \#6 versus Ferrets \#7 and $\# 8$ against $\mathrm{V}_{4}$ (Figure 4E-4H). Interestingly, Ferrets \#5 and \#6 with higher $\mathrm{V}_{4}$ cross-reactive titers after the first infection (left column of Figure 4E-4F) developed lower HAI titers following $\mathrm{V}_{3} \rightarrow \mathrm{V}_{4}$. This can be seen on the neutralization maps, where the purple $\mathrm{V}_{4}$ virus lies within the 
gray circles signifying strong inhibition following $V_{3}$ infection but not following $V_{3} \rightarrow V_{4}$ (Figure 4E-4F). In contrast, Ferrets \#7 and \#8 which started with lower $\mathrm{V}_{4}$ cross-reactive titers developed higher titers following $V_{3} \rightarrow V_{4}$. On the maps, this correspond to $V_{4}$ transitioning from being outside the gray regions to inside them following $\mathrm{V}_{3} \rightarrow \mathrm{V}_{4}$ (Figure $4 \mathrm{G}-4 \mathrm{H}$ ). These results show that while repeated infections can result in robust HAI antibody responses against all infecting strains (Ferrets \#1-4, 7-8), more complex inhibition patterns may arise (Ferrets \#5-6).
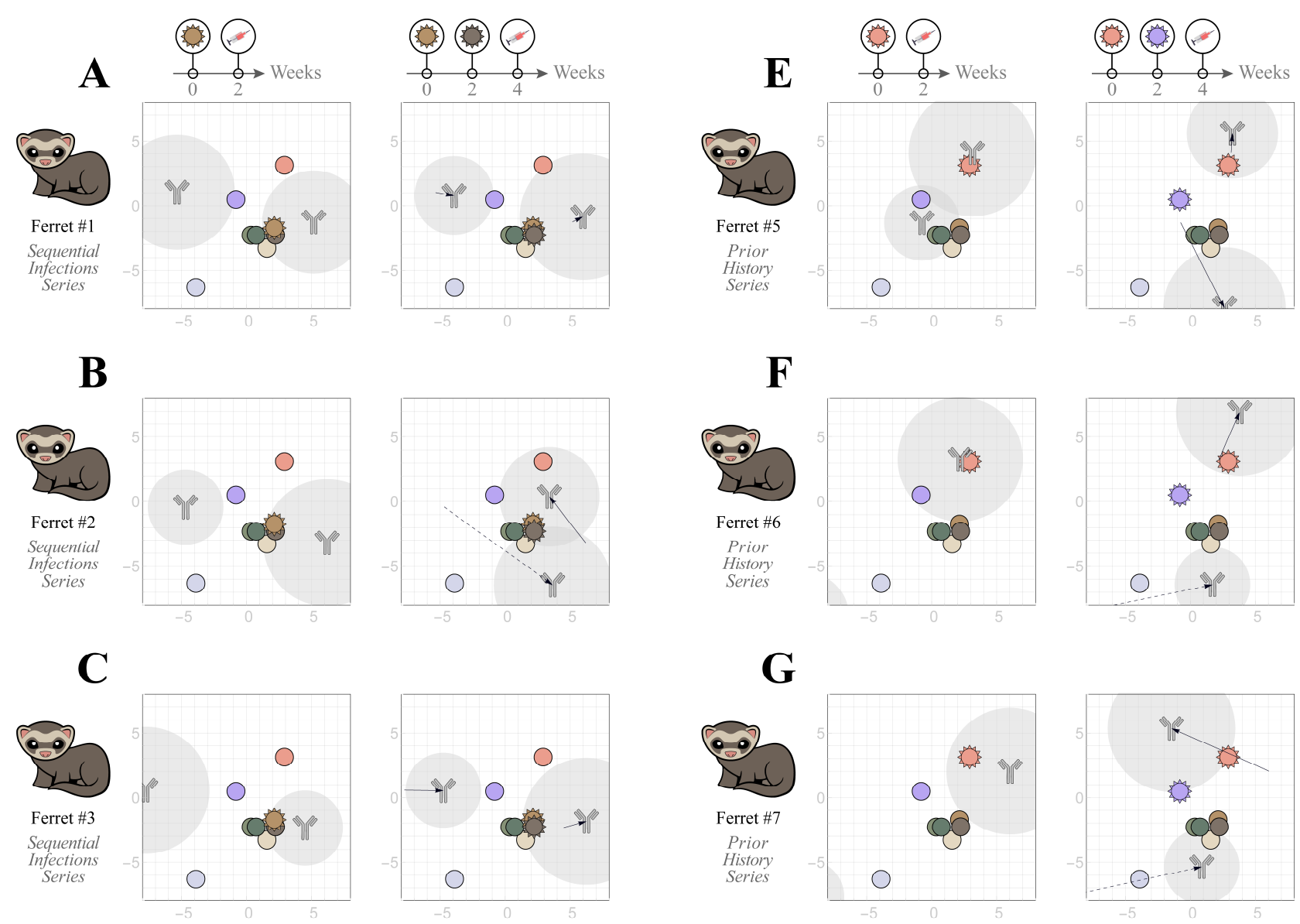

\section{D}
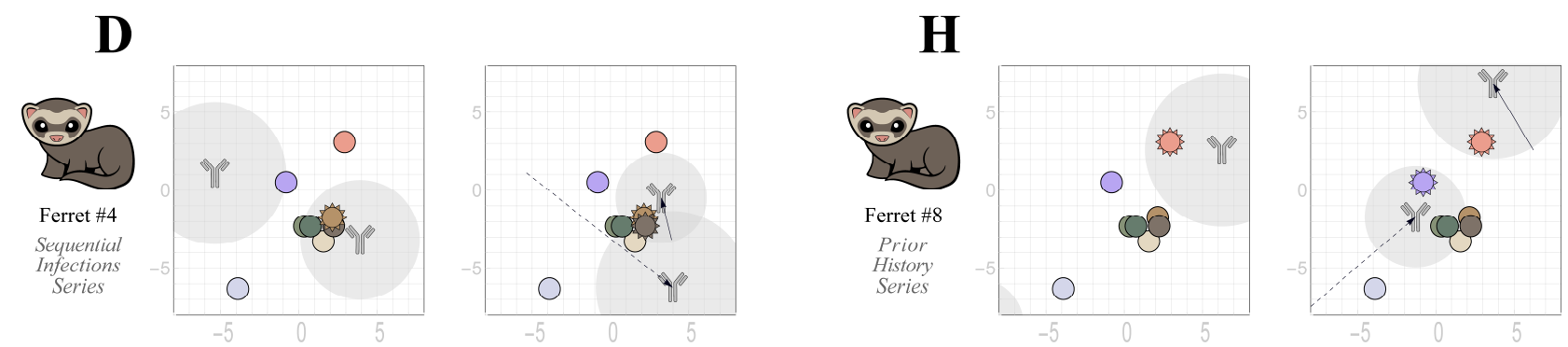

Figure 4. Individual ferret responses after two sequential infections. (A-D) Four ferrets were infected with $\mathrm{V}_{1}=$ Uruguay 2007 followed by $\mathrm{V}_{2}=$ Texas 2012 (E-H) Another group of four ferrets were infected with $\mathrm{V}_{3}=$ Switzerland 2013 followed by $\mathrm{V}_{4}=$ Hong Kong 2014 . In each case, we decompose the antibody response after the first infection and track how each antibody changes after the second infection. Arrows with solid lines indicated the movement of an antibody signature along the map, with long dashed arrows denoting movement greater than 10 grid units that may represent multiple antibodies at the limits of detection [SI Methods]. 


\section{Discussion}

It is estimated that most humans are infected with influenza by the age of 3 and continue to be reinfected by antigenically drifted strains every $5-10$ years. ${ }^{31,32}$ Given the variability in infection histories and the stochastic processes involved in each specific infection, it is exceedingly difficult to determine the composition of pre-existing immunity and how it affects an individual's antibody repertoire. In this study, we combined a ferret reinfection model with newly developed Neutralization Map to dissect the collective antibody response and characterize the properties of the constituent antibodies within. ${ }^{30}$ Using four recent H3N2 vaccine strains Uruguay $2007\left(\mathrm{~V}_{1}\right)$ from the 2008-2010 seasons, Texas $2012\left(\mathrm{~V}_{2}\right)$ from 2013-2015, Switzerland $2013\left(\mathrm{~V}_{3}\right)$ from 2015-2016, and Hong Kong $2014\left(\mathrm{~V}_{4}\right)$ from 2016-2018 - we tracked the antibody footprints through each step of the sequential infections $\mathrm{V}_{1} \rightarrow \mathrm{V}_{2} \rightarrow \mathrm{V}_{3} \rightarrow \mathrm{V}_{4}$ and deciphered the influence of prior exposures on the antibody response under four scenarios $\left(\mathrm{V}_{4}\right.$ alone, $\mathrm{V}_{3} \rightarrow \mathrm{V}_{4}, \mathrm{~V}_{2} \rightarrow \mathrm{V}_{3} \rightarrow \mathrm{V}_{4}$, and $\left.\mathrm{V}_{1} \rightarrow \mathrm{V}_{2} \rightarrow \mathrm{V}_{3} \rightarrow \mathrm{V}_{4}\right)$.

For each infection scheme, we found that the antibody repertoire contains at least one "specific" antibody signature that strongly inhibited the homologous virus and at least one "non-specific" antibody signature that weakly interacted with other $\mathrm{H} 3$ viruses in the panel. Along each step of the exposures $\mathrm{V}_{1} \rightarrow \mathrm{V}_{2} \rightarrow \mathrm{V}_{3} \rightarrow \mathrm{V}_{4}$, both the specific and non-specific signatures tended to move closer to the infection strains. Eventually, a single cross-reactive antibody signature emerged that potently inhibited all four infection strains and the rest of the virus panel except Philippines 1982 (Figure 1H). These results likely reflect that with each infection, antibodies are refocused on conserved epitopes or structural regions across different infection strains. ${ }^{33-35}$

In a classical immune response to the same antigen, the reaction from the primary exposure is greatly magnified in subsequent encounters. OAS occurs upon exposure to antigenically-related viruses, where the response is skewed more heavily towards the earlier infection strains than to the latest strain. While this OAS phenomenon has been suggested to negatively affect vaccine effectiveness, ${ }^{9,18,27,28,36,37}$ seasonal vaccination can persistently extend the number of strains that the human antibody repertoire potently inhibits even when antibodies against earlier viruses are back-boosted. ${ }^{38}$ In this study, we observed OAS with each subsequent infection, regardless of the total number of exposures (Figures 1B-1D and 2B-2D). Despite the ubiquity of OAS, the crossreactivity of ferret antisera increased with each additional infection. These results build upon previous work which suggests that repeated exposures enhance antibody avidity. ${ }^{6}$

In general, repeated exposures resulted in antisera that strongly inhibited all infection strains. The sole exception was that 2 out of 4 ferrets had high $\mathrm{V}_{4}$ cross-reactive HAI titers in the preceding infection (with $\mathrm{V}_{3}$ alone, see left column of Figure 4E-4F) but exhibited a very weak $\mathrm{HAI}$ response towards $\mathrm{V}_{4}$ after infection by $\mathrm{V}_{3} \rightarrow \mathrm{V}_{4}$. In contrast, the other two ferrets in this cohort as well as all four ferrets infected by $\mathrm{V}_{1} \rightarrow \mathrm{V}_{2}$, elicited the typical response where both infection strains were strongly inhibited (Figure 4A-4D, 4G-4H). While it is unclear whether biological variation drives these observed differences, these results demonstrate that sequential infection can alter the patterns of an antibody response. 
One feature of the neutralization maps is that the positions of the eight viruses in the panel were determined using data for human monoclonal antibodies, which may differ from ferret postinfection antisera. One such discrepancy is that the antigenic distance between $\mathrm{V}_{1}$ (Uruguay 2007) and $V_{2}$ (Texas 2012) is small according to human monoclonal antibodies ${ }^{39}$ and postvaccination human sera, ${ }^{3}$ but that both viruses are considered antigenically distinct using ferret antisera raised from a single infection. ${ }^{3}$ In this study, substantially different HAI titers were observed across these two strains (Figures 1A-1B, 2A-2C, 3A-3B), which confirms that the ferret immune system treats $\mathrm{V}_{1}$ and $\mathrm{V}_{2}$ as antigenically distinct. Nevertheless, the full suite of HAI titers presented on the maps shows an average 2-fold error to the experimental measurements (Figures S1, S2C), demonstrating that the antigenic relationships among the majority of the viruses in the panel are the same across humans and ferrets.

In summary, by tracking the changes in the inhibition profile of ferret antisera induced by repeated influenza $\mathrm{A} / \mathrm{H} 3$ infections, we demonstrated that an antibody could be guided along the map after a series of infections and that prior immune history can heavily influence the ferret antibody response. Despite the presence of OAS in each ferret infected by two or more viruses, a broadly neutralizing antibody signature that potently inhibited all infection strains was nevertheless produced (Figure $1 \mathrm{H}$ ). While our current work was focused on HA head-specific antibodies, it does not consider antibodies directed towards the HA stem and neuraminidase that have also been shown to exhibit OAS and may influence the dynamics of this system. ${ }^{40,41}$ Future work that refines these antibody trajectories across multiple infections and multiple regions of an influenza virus may facilitate the development of more effective influenza vaccines.

\section{Methods}

Viruses

The panel of H3N2 viruses used for the study included A/Philippines/2/1982 (Philippine 1982), A/Wisconsin/67/2005 (Wisconsin 2005), A/Uruguay/10/2007 (Uruguay 2007), A/Perth/16/2009 (Perth 2009), A/Victoria/361/2011 (Victoria 2011), A/Texas/50/2012 (Texas 2012), A/Switzerland/9715293/2013 (Switzerland 2013) and A/Hong Kong/4801/2014 (Hong Kong 2014), each of which has served as the prototype for the H3N2 seasonal influenza vaccine component in the past decades. All H3N2 viruses were propagated in 9-10 days old embryonated eggs and aliquots were stored at $-80^{\circ} \mathrm{C}$ until use.

\section{Ferret Infection}

Seronegative male ferrets (Triple F Farm) at 15-16 weeks old were infected intranasally at twoweek intervals with each of the four $\mathrm{H} 3 \mathrm{~N} 2$ viruses $\left(\mathrm{V}_{1}=\mathrm{A} / \mathrm{Uruguay} / 10 / 2007\right.$, $\mathrm{V}_{2}=\mathrm{A} /$ Texas/50/2012, $\mathrm{V}_{3}=\mathrm{A} / \mathrm{Switzerland} / 9715293 / 2013$, and $\mathrm{V}_{4}=\mathrm{A} /$ Hong Kong/4801/2014) as previously reported. ${ }^{6}$ Ferrets were bled via venipuncture of the cranial vena cava at 14 days after each infection. Sera from four ferrets in each infection scheme were collected for HAI titer determination. All procedures were carried out in accordance with a protocol approved by the 
Institutional Animal Care and Use Committee of the Center for Biologics Evaluation and Research, US Food and Drug Administration.

\section{HAI Assay}

Following pre-treatment with a receptor-destroying enzyme (Denka-Seiken), individual ferret sera were 2-fold serially diluted and were $1: 1(\mathrm{v} / \mathrm{v})$ incubated with testing virus solution containing 4 hemagglutinin (HA) units per $25 \mu \mathrm{L}$ at room temperature for $30 \mathrm{~min}$ before the addition of $50 \mu \mathrm{L}$ of $0.75 \%$ guinea pig erythrocytes in the presence of $20 \mathrm{nM}$ oseltamivir as previously described. ${ }^{6,14}$ The endpoint HAI titer was defined as the reciprocal of the highest serum dilution that yielded a complete HA inhibition, and a titer 5 was assigned if no inhibition was observed at the starting 1:10 serum dilution. HAI geometric mean titers (GMTs) were calculated along with the $95 \%$ confidence intervals.

\section{$\underline{\text { Decomposing Ferret Antisera on the Neutralization Maps }}$}

The positions of the eight H3N2 viruses in the testing panel were previously determined using neutralizing titers from 6 human monoclonal antibodies. ${ }^{30}$ These maps quantify how an individual antibody simultaneously inhibits all eight $\mathrm{H} 3 \mathrm{~N} 2$ viruses, where an antibody-virus distance $d$ on the map corresponds to an HAI titer of 3000/2 ${ }^{d}$. A key assumption of this analysis is that each antibody can be represented by a single point on the map, so that scanning the map exhaustively searches through the possible inhibition profiles for any antibody targeting the head domain of influenza hemagglutinin.

To decompose each serum, we searched through an increasing number of antibodies to determine which combination of coordinates and stoichiometries best matched the HAI titers of the eight H3N2 viruses in the panel [SI Methods]. The process ended when adding an additional antibody did not substantially improve the match with the experimental measurements. We validated the resulting decompositions in two ways. First, we determined that the HAI titers presented on the maps were on average only 2-fold off from the experimental measurements, demonstrating that these maps can capture the trends in the HAI profiles (Figure S1). Next, we decomposed each antiserum using HAI titers from only four viruses (Philippines 1982, Wisconsin 2005, Switzerland 2013, Hong Kong 2014). The predicted titers against the remaining four viruses were only 5-fold off from the experimental measurements, comparable to the results from earlier work analyzing mixtures with known antibody composition (Figure S2). ${ }^{30}$

\section{Funding}

This work was supported by the intramural research fund of Center for Biologics Evaluation and Research, US Food and Drug Administration. Tal Einav is a Damon Runyon Fellow supported by the Damon Runyon Cancer Research Foundation (DRQ 01-20).

\section{Author contributions}

$\mathrm{H}$ Xie conceived and designed the ferret study. M Kosikova, P Radvak, H Xie, and Y-C Kuo 
conducted the ferret infection experiments. M Kosikova, H Xie, P Radvak and H J Kwon performed the HAI assays. T Einav conceived and designed Neutralization Map. T Einav, M Kosikova and $\mathrm{H}$ Xie analyzed the HAI data. H Xie and T Einav wrote the paper.

\section{Disclaimer}

The findings and conclusions in this article have not been formally disseminated by US Food and Drug Administration and should not be construed to represent any Agency determination or policy.

\section{References}

1 Belongia, E. A. et al. Effectiveness of inactivated influenza vaccines varied substantially with antigenic match from the 2004-2005 season to the 2006-2007 season. J Infect Dis 199, 159-167, doi:10.1086/595861 (2009).

2 Skowronski, D. M. et al. Low 2012-13 influenza vaccine effectiveness associated with mutation in the egg-adapted H3N2 vaccine strain not antigenic drift in circulating viruses. PLoS One 9, e92153, doi:10.1371/journal.pone.0092153 [doi];PONE-D-13-54409 [pii] (2014).

3 Xie, H. et al. H3N2 Mismatch of 2014-15 Northern Hemisphere Influenza Vaccines and Head-to-head Comparison between Human and Ferret Antisera derived Antigenic Maps. Sci Rep 5, 15279, doi:10.1038/srep15279 (2015).

4 Flannery, B. et al. Early estimates of seasonal influenza vaccine effectiveness - United States, January 2015. MMWR Morb Mortal. Wkly Rep 64, 10-15, doi:mm6401a4 [pii] (2015).

5 Treanor, J. J. et al. Effectiveness of seasonal influenza vaccines in the United States during a season with circulation of all three vaccine strains. Clin Infect Dis 55, 951-959, doi:10.1093/cid/cis574 (2012).

6 Kosikova, M. et al. Imprinting of Repeated Influenza A/H3 Exposures on Antibody Quantity and Antibody Quality: Implications for Seasonal Vaccine Strain Selection and Vaccine Performance. Clin Infect Dis 67, 15231532, doi:10.1093/cid/ciy327 (2018).

7 Treanor, J. What Happens Next Depends on What Happened First. Clin Infect Dis 67, 1533-1534, doi:10.1093/cid/ciy330 (2018).

8 Henry, C., Palm, A. E., Krammer, F. \& Wilson, P. C. From Original Antigenic Sin to the Universal Influenza Virus Vaccine. Trends in immunology 39, 70-79, doi:10.1016/j.it.2017.08.003 (2018).

9 Monto, A. S., Malosh, R. E., Petrie, J. G. \& Martin, E. T. The Doctrine of Original Antigenic Sin: Separating Good From Evil. J Infect Dis 215, 1782-1788, doi:10.1093/infdis/jix173 (2017).

$10 \mathrm{Liu}, \mathrm{F}$. et al. Influence of Immune Priming and Egg Adaptation in the Vaccine on Antibody Responses to Circulating A(H1N1)pdm09 Viruses After Influenza Vaccination in Adults. J Infect Dis 218, 1571-1581, doi:10.1093/infdis/jiy376 (2018).

11 Andrews, S. F. et al. High preexisting serological antibody levels correlate with diversification of the influenza vaccine response. $J$ Virol 89, 3308-3317, doi:10.1128/JVI.02871-14 (2015).

12 Andrews, S. F. et al. Immune history profoundly affects broadly protective B cell responses to influenza. Sci Transl Med 7, 316ra192, doi:10.1126/scitranslmed.aad0522 (2015).

$13 \mathrm{Li}$, Y. et al. Immune history shapes specificity of pandemic H1N1 influenza antibody responses. J Exp. Med 210, 1493-1500, doi:jem.20130212 [pii];10.1084/jem.20130212 [doi] (2013).

14 Xie, H. et al. Differential Effects of Prior Influenza Exposures on H3N2 Cross-reactivity of Human Postvaccination Sera. Clin Infect Dis 65, 259-267, doi:10.1093/cid/cix269 (2017).

$15 \mathrm{Xie}$, H. et al. Immunogenicity and cross-reactivity of 2009-2010 inactivated seasonal influenza vaccine in US adults and elderly. PLoS One 6, e16650, doi:10.1371/journal.pone.0016650 (2011).

$16 \mathrm{Xie}, \mathrm{H}$. et al. Revisiting the 1976 "swine flu" vaccine clinical trials: cross-reactive hemagglutinin and neuraminidase antibodies and their role in protection against the $2009 \mathrm{H} 1 \mathrm{~N} 1$ pandemic virus in mice. Clin. Infect. Dis 53, 1179-1187, doi:cir693;10.1093/cid/cir693 (2011).

17 Linderman, S. L. et al. Potential antigenic explanation for atypical H1N1 infections among middle-aged adults during the 2013-2014 influenza season. Proc. Natl. Acad. Sci U S. A 111, 15798-15803, doi:1409171111 [pii];10.1073/pnas.1409171111 [doi] (2014).

18 Choi, Y. S. et al. Reduced antibody responses to the pandemic (H1N1) 2009 vaccine after recent seasonal influenza vaccination. Clin Vaccine Immunol 18, 1519-1523, doi:10.1128/CVI.05053-11 (2011). 
19 Sasaki, S. et al. Influence of prior influenza vaccination on antibody and B-cell responses. PLoS One 3, e2975, doi:10.1371/journal.pone.0002975 (2008).

20 McLean, H. Q. et al. Impact of repeated vaccination on vaccine effectiveness against influenza A(H3N2) and B during 8 seasons. Clin Infect Dis 59, 1375-1385, doi:10.1093/cid/ciu680 (2014).

21 Ohmit, S. E. et al. Influenza vaccine effectiveness in households with children during the 2012-2013 season: assessments of prior vaccination and serologic susceptibility. J Infect Dis 211, 1519-1528, doi:10.1093/infdis/jiu650 (2015).

22 Ohmit, S. E. et al. Influenza vaccine effectiveness in the 2011-2012 season: protection against each circulating virus and the effect of prior vaccination on estimates. Clin Infect Dis 58, 319-327, doi:10.1093/cid/cit736 (2014).

23 Saito, N. et al. Negative impact of prior influenza vaccination on current influenza vaccination among people infected and not infected in prior season: A test-negative case-control study in Japan. Vaccine 35, 687-693, doi:10.1016/j.vaccine.2016.11.024 (2017).

24 Rondy, M. et al. Repeated seasonal influenza vaccination among elderly in Europe: Effects on laboratory confirmed hospitalised influenza. Vaccine 35, 4298-4306, doi:10.1016/j.vaccine.2017.06.088 (2017).

25 Skowronski, D. M. et al. Serial Vaccination and the Antigenic Distance Hypothesis: Effects on Influenza Vaccine Effectiveness During A(H3N2) Epidemics in Canada, 2010-2011 to 2014-2015. J Infect Dis 215, 1059-1099, doi:10.1093/infdis/jix074 (2017).

26 Francis, T., Jr. . On the Doctrine of Original Antigenic Sin. Proceedings of the American Philosophical Society 104, 572-578 (1960).

27 Lewnard, J. A. \& Cobey, S. Immune History and Influenza Vaccine Effectiveness. Vaccines (Basel) 6, doi:10.3390/vaccines6020028 (2018).

28 Yewdell, J. W. \& Santos, J. J. S. Original Antigenic Sin: How Original? How Sinful? Cold Spring Harb Perspect Med, doi:10.1101/cshperspect.a038786 (2020).

29 Zhang, A., Stacey, H. D., Mullarkey, C. E. \& Miller, M. S. Original Antigenic Sin: How First Exposure Shapes Lifelong Anti-Influenza Virus Immune Responses. J Immunol 202, 335-340, doi:10.4049/jimmunol.1801149 (2019).

30 Einav, T., Creanga, A. \& Kanekiyo, M. Decomposing Polyclonal Sera using Neutralization Maps. bioRxiv, doi:https://doi.org/10.1101/2020.08.28.270561 (2020).

31 Bodewes, R. et al. Prevalence of antibodies against seasonal influenza A and B viruses in children in Netherlands. Clin Vaccine Immunol 18, 469-476, doi:10.1128/CVI.00396-10 (2011).

32 Kucharski, A. J. et al. Estimating the Life Course of Influenza A(H3N2) Antibody Responses from Cross-Sectional Data. PLoS Biol 13, e1002082, doi:10.1371/journal.pbio.1002082;PBIOLOGY-D-14-01366 (2015).

33 Sahini, L., Tempczyk-Russell, A. \& Agarwal, R. Large-scale sequence analysis of hemagglutinin of influenza A virus identifies conserved regions suitable for targeting an anti-viral response. PLoS One 5, e9268, doi:10.1371/journal.pone.0009268 (2010).

34 Wong, T. M. et al. Computationally Optimized Broadly Reactive Hemagglutinin Elicits Hemagglutination Inhibition Antibodies against a Panel of H3N2 Influenza Virus Cocirculating Variants. J Virol 91, doi:10.1128/JVI.01581-17 (2017).

$35 \mathrm{Wu}, \mathrm{K} . \mathrm{W}$., Chien, C. Y., Li, S. W., King, C. C. \& Chang, C. H. Highly conserved influenza A virus epitope sequences as candidates of H3N2 flu vaccine targets. Genomics 100, 102-109, doi:10.1016/j.ygeno.2012.06.003 (2012).

36 Skowronski, D. M. et al. Beyond Antigenic Match: Possible Agent-Host and Immuno-epidemiological Influences on Influenza Vaccine Effectiveness During the 2015-2016 Season in Canada. J Infect Dis 216, 1487-1500, doi:10.1093/infdis/jix526 (2017).

37 Smith, D. J., Forrest, S., Ackley, D. H. \& Perelson, A. S. Variable efficacy of repeated annual influenza vaccination. Proc. Natl. Acad. Sci U. S. A 96, 14001-14006 (1999).

38 Fonville, J. M. et al. Antibody landscapes after influenza virus infection or vaccination. Science 346, 996-1000, doi:346/6212/996 [pii];10.1126/science.1256427 [doi] (2014).

39 Creanga, A. et al. A comprehensive influenza reporter virus panel for high-throughput deep profiling of neutralizing antibodies. bioRxiv, doi:https://doi.org/10.1101/2020.02.24.963611 (2020).

40 Arevalo, C. P. et al. Original antigenic sin priming of influenza virus hemagglutinin stalk antibodies. Proc Natl Acad Sci U S A 117, 17221-17227, doi:10.1073/pnas.1920321117 (2020).

41 Rajendran, M. et al. Analysis of Anti-Influenza Virus Neuraminidase Antibodies in Children, Adults, and the Elderly by ELISA and Enzyme Inhibition: Evidence for Original Antigenic Sin. mBio 8, doi:10.1128/mBio.0228116 (2017). 